\title{
Extension of Friedel's law to vortex-beam diffraction
}

\author{
Roeland Juchtmans, Giulio Guzzinati, and Jo Verbeeck \\ EMAT, University of Antwerp, Groenenborgerlaan 171, 2020 Antwerp, Belgium
}

(Received 23 August 2016; published 30 September 2016)

\begin{abstract}
Friedel's law states that the modulus of the Fourier transform of real functions is centrosymmetric, while the phase is antisymmetric. As a consequence of this, elastic scattering of plane-wave photons or electrons within the first-order Born-approximation, as well as Fraunhofer diffraction on any aperture, is bound to result in centrosymmetric diffraction patterns. Friedel's law, however, does not apply for vortex beams, and centrosymmetry in general is not present in their diffraction patterns. In this work we extend Friedel's law for vortex beams by showing that the diffraction patterns of vortex beams with opposite topological charge, scattered on the same two-dimensional potential, always are centrosymmetric to one another, regardless of the symmetry of the scattering object. We verify our statement by means of numerical simulations and experimental data. Our research provides deeper understanding in vortex-beam diffraction and can be used to design new experiments to measure the topological charge of vortex beams with diffraction gratings or to study general vortex-beam diffraction.
\end{abstract}

DOI: 10.1103/PhysRevA.94.033858

\section{INTRODUCTION}

Friedel's law (FL) states that two antisymmetric points in the Fourier transform of a real function $V(\boldsymbol{r})$ are complex conjugated to one another, $\mathcal{F}[V](\boldsymbol{k})=\mathcal{F}^{*}[V](-\boldsymbol{k})$. By deriving this rule, the French crystallographer George Friedel was able to explain why, within the kinematical approximation, the zeroth-order Laue zone in X-ray diffraction patterns always is centrosymmetric and showed how this puts restrictions on the possible crystal symmetries that can be directly determined by x-ray diffraction [1]. Not only does FL apply to x-ray diffraction on crystals, it generally is valid for all scattering processes where a plane wave scatters on (approximately) twodimensional objects that are described by a real transmittance function, such as apertures or gratings. Whereas for electron diffraction on crystals FL breaks down relatively fast because of dynamical scattering, it remains valid for electrons scattered on apertures. Since FL is valid only for plane waves, the diffraction patterns of distorted or modified beams, like the so-called vortex beams, do not necessarily follow this rule.

Vortex beams are eigenstates of the orbital angular momentum (OAM) operator, $\hat{L}_{z}=-i \hbar \frac{\partial}{\partial \phi}[2]$, and their wave function has the form

$$
\Psi_{m}(\boldsymbol{r})=\psi(r, z) e^{i \ell \phi},
$$

with $r$ and $\phi$ being the radial and the azimuthal coordinates with respect to the wave-propagation axis $z$. The number $\ell$ is called the topological charge (TC) of the vortex. Since vortex beams are eigenstates of the angular momentum operator, they possess a well-defined angular momentum of $\ell \hbar$ per photon [3], electron [4], or any other particle [5] described by the wave function in Eq. (1). These beams have a typical donutlike intensity profile, a bright ring with a black spot in the middle, that arises from the phase singularity at its center. Since their first experimental demonstration in optics [6], they have been studied extensively, both theoretically and experimentally, which has led to numerous applications in fields such as nanomanipulation [7-9], astrophysics [10-13], and telecommunications [14-16]. Although slightly more complicated, a vast number of methods have been developed to produce vortex beams in an electron microscope [17-20] as well, and several studies suggest their use to probe magnetism [18,21], in nanomanipulation [22], in spin-polarization devices [23], and in measuring the chirality of crystals [24]. Additionally, the expression in Eq. (1) provides an excellent mathematical basis to describe scattering on helical crystals [25] or processes involving local exchange of OAM [26].

Many of the above applications require an accurate measurement of the TC of the vortex beam. A popular way of doing this is to study the vortex diffraction patterns of specially designed apertures such as triangular apertures [27,28] or multipinhole plates [29-31]. In this work we elaborate on the observation made in these studies that diffraction patterns of vortex beams with opposite TC always seem to be centrosymmetric, or, equivalently, rotated $\pi \mathrm{rad}$, with respect to each other, independent of the shape of the aperture. Although in the above research this can be explained using the symmetry of these specific apertures, we will formulate an extension of FL as a more fundamental property of vortexbeam diffraction. We illustrate with numerical simulations and experiments that even for nonsymmetric scattering processes, the twofold rotational symmetry between diffraction patterns with oppositely charged vortex beams remains present. This research provides further insights into vortex-beam diffraction and can help to optimize methods to measure the TC of vortex beams using diffraction gratings or to study general vortex-beam diffraction.

\section{THEORETICAL FORMULATION}

\section{A. Friedel's law for plane waves}

Friedel's law includes two properties of the Fourier transform (FT) of real functions [1].

Given a real function $f(\boldsymbol{r})$, its FT is given by

$$
\mathcal{F}(\boldsymbol{k})=\int d \boldsymbol{r} f(\boldsymbol{r}) e^{i \boldsymbol{k} \cdot \boldsymbol{r}}
$$


Looking at the complex conjugate of the FT,

$$
\begin{aligned}
{[\mathcal{F}(\boldsymbol{k})]^{*} } & =\left[\int d \boldsymbol{r} f(\boldsymbol{r}) e^{i \boldsymbol{k} \cdot \boldsymbol{r}}\right]^{*} \\
& =\int d \boldsymbol{r} f(\boldsymbol{r}) e^{-i \boldsymbol{k} \cdot \boldsymbol{r}}=\mathcal{F}(-\boldsymbol{k}),
\end{aligned}
$$

one can easily see that the modulus of the FT is centrosymmetric,

$$
|F(\boldsymbol{k})|=|F(-\boldsymbol{k})|,
$$

while its phase $\phi(\boldsymbol{k})$ is antisymmetric,

$$
\phi(\boldsymbol{k})=-\phi(\boldsymbol{k}) .
$$

Equations (4) and (5) are known as Friedel's law, and any set of centrosymmetric points $(\boldsymbol{k},-\boldsymbol{k})$ is called a Friedel pair.

Friedel applied these properties to describe the symmetries seen in x-ray diffraction patterns in crystals. For a scalar plane-wave photon that is scattered kinematically by a crystal, the scattering potential is a real function. Within the single-scattering approximation, valid for weakly interacting samples, the scattering amplitude of a plane-wave photon, with wave number $\boldsymbol{k}$, to scatter to a plane wave with wave number $\boldsymbol{k}^{\prime}$ then is given by

$$
\begin{aligned}
A\left(\boldsymbol{k}, \boldsymbol{k}^{\prime}\right) & =\left\langle\boldsymbol{k}^{\prime}|V(\boldsymbol{r})| \boldsymbol{k}\right\rangle=\int d \boldsymbol{r} e^{-i \boldsymbol{k}^{\prime} \cdot \boldsymbol{r}} V(\boldsymbol{r}) e^{i \boldsymbol{k} \cdot \boldsymbol{r}} \\
& =\int d \boldsymbol{r} V(\boldsymbol{r}) e^{i \Delta \boldsymbol{k} \cdot \boldsymbol{r}},
\end{aligned}
$$

with $\Delta \boldsymbol{k}=\boldsymbol{k}-\boldsymbol{k}^{\prime}$. Clearly, the scattering amplitude exactly equals the FT of the potential. The X-ray diffraction pattern is given by the intersection of the three-dimensional scattering amplitude with the so-called Ewald sphere, which ensures that the length of the wave vector of the outgoing photon, and thus the energy, is the same as that of the incoming photon [32]. In general, the curvature of this sphere can be considered flat for any relevant scattering angle, and the scattering amplitude is determined by the two-dimensional FT of the potential projected along the $z$ axis, the photon's propagation axis, $V_{\perp}\left(\boldsymbol{r}_{\perp}\right)=\int d z V(x, y, z)$,

$$
A\left(\boldsymbol{k}, \boldsymbol{k}^{\prime}\right)=\int d \boldsymbol{r}_{\perp} V_{\perp}\left(\boldsymbol{r}_{\perp}\right) e^{i \boldsymbol{k}_{\perp}^{\prime} \cdot \boldsymbol{r}_{\perp}}
$$

where, here and in the following, $\boldsymbol{r}_{\perp}$ denotes the twodimensional coordinate in the $(x, y)$ plane. The diffraction patterns in the kinematical approximation, given by the scattering amplitude squared $\left|A\left(\boldsymbol{k}, \boldsymbol{k}^{\prime}\right)\right|^{2}$, will be centrosymmetric because of FL, making it impossible to distinguish certain symmetries in the projection of crystals with one single diffraction pattern. A crystal with a threefold rotation axis along the projection direction, for instance, will show the same symmetry in its diffraction pattern as a crystal with a sixfold rotation axis [1].

Besides x-ray diffraction on crystals, FL also applies to diffraction of plane waves on apertures and gratings. Consider, for instance, an aperture in the $x y$ plane that is placed at $z_{0}=0$, illuminated by a plane wave of the form $\Psi_{0}=e^{i k_{z} z_{0}}$. The diffracted wave in the far field of the aperture then is given
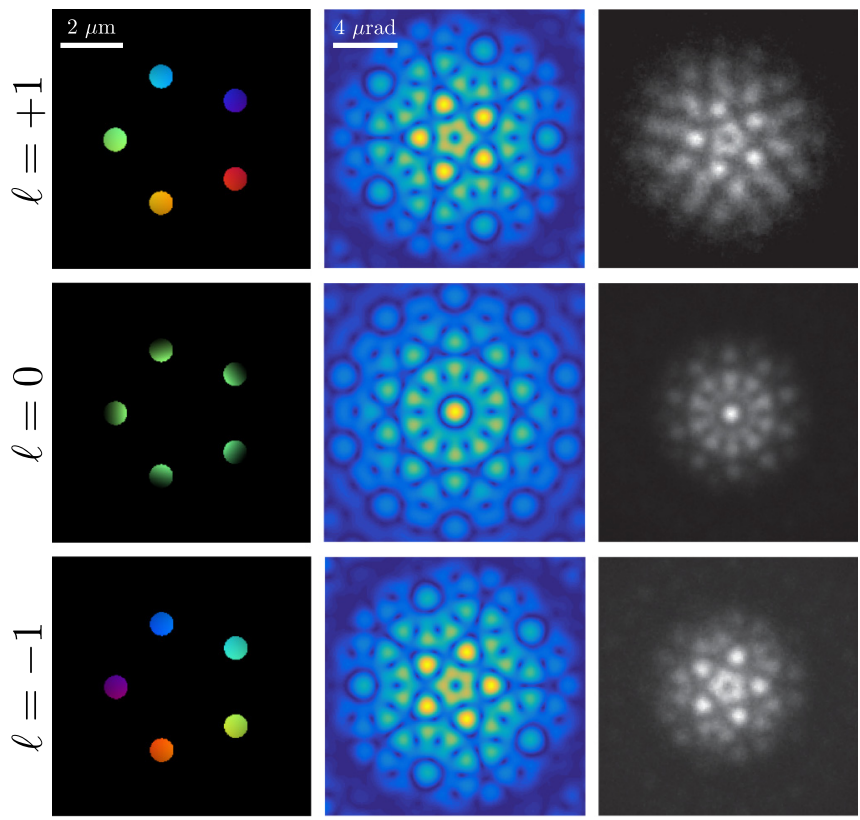

FIG. 1. (Left) Illumination of a fivefold multipinhole aperture with an $\ell=-1, \ell=0$, and $\ell=1$ vortex, as in [29] and [31]. (Middle) Simulated diffraction pattern. (Right) Experimental diffraction pattern. Since the axis of the vortex lies on the fivefold symmetry axis of the aperture, all diffraction patterns show the fivefold symmetry of the pinhole aperture. The $\ell=0$ diffraction pattern is twofold rotation symmetric as well, demonstrating the conventional Friedel's law. This symmetry is absent in the $\ell=-1$ and $\ell=1$ diffraction patterns, but as expected from Eq. (11), they do show twofold rotational symmetry with respect to each other.

by the Fraunhofer equation [33]

$$
\begin{aligned}
\Psi\left(\boldsymbol{k}_{\perp}, z\right) & =\int d \boldsymbol{r}_{\perp}^{\prime} f\left(\boldsymbol{r}_{\perp}^{\prime}\right) e^{i \frac{k_{z}}{2} \boldsymbol{r}_{\perp} \cdot \boldsymbol{r}_{\perp}^{\prime}} \Psi\left(\boldsymbol{r}_{\perp}^{\prime}, 0\right) \\
& =\int d \boldsymbol{k}_{\perp}^{\prime} f\left(\boldsymbol{r}_{\perp}^{\prime}\right) e^{i \frac{k_{z}}{2} \boldsymbol{r}_{\perp} \cdot \boldsymbol{r}_{\perp}^{\prime}} \\
& =\int d \boldsymbol{k}_{\perp}^{\prime} f\left(\boldsymbol{r}_{\perp}^{\prime}\right) e^{i \boldsymbol{k}_{\perp} \cdot \boldsymbol{r}_{\perp}^{\prime}},
\end{aligned}
$$

with $\boldsymbol{k}_{\perp}=\frac{k_{z}}{z} \boldsymbol{r}_{\perp}^{\prime}, z$ being the distance from the aperture, and $f\left(x^{\prime}, y^{\prime}\right)$ being the transmittance function of the grating. In general $f\left(r_{\perp}^{\prime}\right)$ does not have to be real. In the case of phase plates, for instance, where the phase of the wave is altered, $f\left(\boldsymbol{r}_{\perp}^{\prime}\right)$ is complex. But for apertures that only change the amplitude, $f\left(\boldsymbol{r}_{\perp}^{\prime}\right)$ is real. In this case it is clear we can apply Friedel's law and the intensity pattern of the diffracted wave will always be centrosymmetric, independent of the symmetry of the diffraction grating. This is shown numerically and experimentally in Fig. 1.

\section{B. Friedel's law for vortex beams}

Friedel's law applies only to diffraction of plane waves. When the incoming beam is modified or distorted, it is no longer valid. This is the case for an incoming vortex beam of 

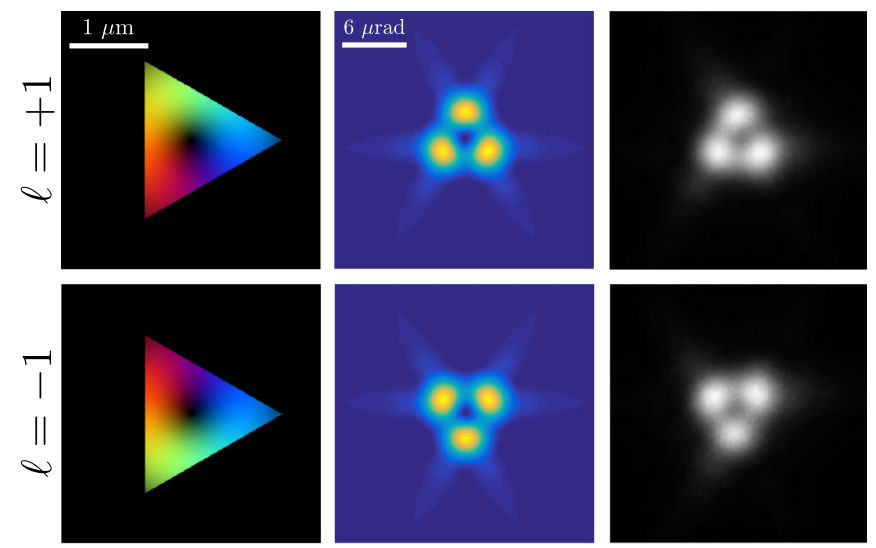

FIG. 2. (Left) Illumination of a triangular aperture with an $\ell=$ -1 and $\ell=1$ vortex, as in [27]. (Middle) Simulated diffraction pattern. (Right) Experimental diffraction pattern. Since the axis of the vortex lies on the threefold symmetry axis of the aperture, all diffraction patterns show threefold symmetry. Again, the $\ell=-1$ and $\ell=1$ diffraction patterns show twofold rotational symmetry with respect to each other.

the form

$$
\Psi_{\ell}(\boldsymbol{r})=\psi_{\ell}(r, z) e^{i \ell \phi}
$$

where $\ell$ is the topological charge and $\psi_{\ell}(r, z)$ is the radial profile of the beam at height $z$. The symmetry of the diffraction pattern of such a beam scattered on an aperture or crystal in general will not be centrosymmetric anymore (see Figs. 1, 2, and 3). However, applying the same trick as in Eq. (3), we can easily find a relation between the diffraction patterns of vortex beams with opposite topological charge having the same real radial profile.

Consider scattering of a vortex beam on an aperture with real transmittance function $f\left(\boldsymbol{r}_{\perp}\right)$ placed at height $z_{0}=0$. Like in Eq. (8), the wave in the far field now is given by the
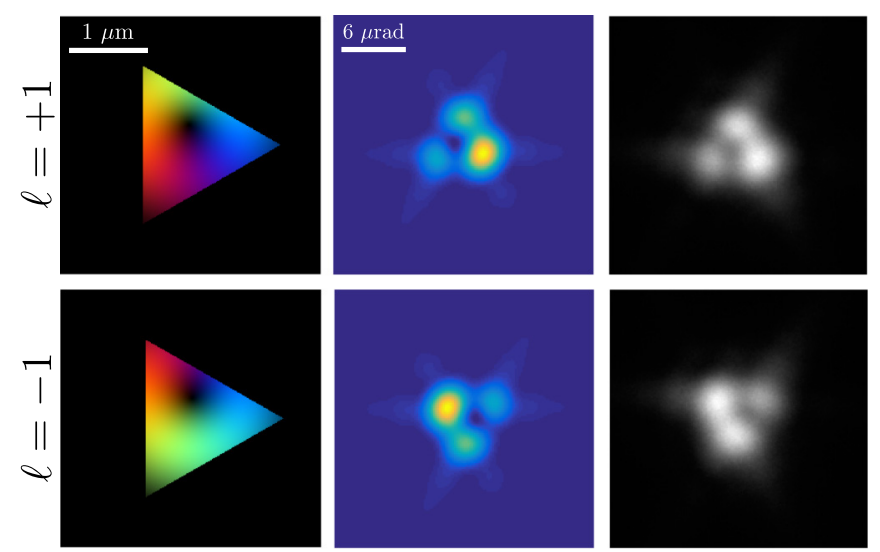

FIG. 3. Same as in Fig. (2), but now the axis of the vortex lies on a nonsymmetric point of the aperture. Therefore the diffraction patterns no longer show the symmetry of the aperture. However, consistent with Eq. (11), the $\ell=-1$ and $\ell=1$ diffraction patterns are rotated $\pi \mathrm{rad}$ with respect to one another. This shows that this effect is not dependent on the symmetry of the scattering object but is a more fundamental effect of vortex scattering.
Fraunhofer equation

$$
\Psi_{\ell}\left(\boldsymbol{r}_{\perp}, z\right)=\int d \boldsymbol{r}_{\perp}^{\prime} f\left(\boldsymbol{r}_{\perp}^{\prime}\right) e^{i \frac{k z}{z} r_{\perp} \cdot r_{\perp}^{\prime}} \Psi_{\ell}\left(\boldsymbol{r}_{\perp}^{\prime}, 0\right) .
$$

When we look at the complex conjugate of the scattered wave, we get

$$
\begin{aligned}
\Psi_{\ell}^{*}\left(\boldsymbol{r}_{\perp}, z\right) & =\left(\int d \boldsymbol{r}_{\perp}^{\prime} f\left(\boldsymbol{r}_{\perp}^{\prime}\right) e^{i \frac{k_{z}}{z} \boldsymbol{r}_{\perp} \cdot \boldsymbol{r}_{\perp}^{\prime}} \Psi_{\ell}\left(\boldsymbol{r}_{\perp}^{\prime}, 0\right)\right)^{*} \\
& =\int d \boldsymbol{r}_{\perp}^{\prime} f\left(\boldsymbol{r}_{\perp}^{\prime}\right) e^{-i \frac{k_{z}}{z} \boldsymbol{r}_{\perp} \cdot \boldsymbol{r}_{\perp}^{\prime}} \psi_{\ell}(r, 0) e^{-i \ell \phi} \\
& =\Psi_{-\ell}\left(-\boldsymbol{r}_{\perp}, z\right) .
\end{aligned}
$$

This means that diffraction patterns of oppositely charged vortex probes with a real radial profile will be centrosymmetric, or rotated $\pi \mathrm{rad}$, with respect to each other. The same goes for diffraction on a crystal when replacing the incoming plane wave in Eq. (6) with a vortex beam. Note that the radial profile of the beam has to be real, as is the case for most typical vortex beams such as the nondiffracting Bessel beams, $\psi_{\ell}^{\mathrm{B}}(r, z)=J_{\ell}(k r)$, or the Laguerre Gaussian beams in the focal plane, $\psi_{\ell}^{\mathrm{LG}}(r, 0)=\left(\frac{r}{k}\right)^{\ell} L_{n}^{\ell}\left(\frac{r^{2}}{k^{2}}\right) \exp \left(-\frac{r^{2}}{k^{2}}\right)$, where $L_{n}^{\ell}$ is the Laguerre polynomial with radial and angular modes $n$ and $\ell$, respectively, and $k$ is a parameter determining the width of the beam. Note that when defocussing a vortex beam with a real radial function, this radial function becomes complex, and Eq. (11) no longer holds.

We will refer to Eq. (11) as the extension of Friedel's law (EFL) applicable for vortex beams. It includes the known Friedel's law when considering a plane wave as a vortex beam with topological charge $\ell=0$.

\section{SIMULATION AND EXPERIMENT}

In this section, we show simulations of vortex-beam diffraction on multipinhole and triangular apertures and compare these with electron vortex-beam experiments on a probecorrected FEI Titan ${ }^{3}$ microscope. The incoming vortex wave with TC $\ell$ numerically is simulated by

$$
\Psi_{\ell}(r, \phi)=\int_{0}^{k_{\max }} d k \int_{0}^{2 \pi} d \phi_{k} e^{i \ell \phi_{k}} e^{i k r \cos \left(\phi_{k}-\phi\right)},
$$

with $k_{\max }=\alpha_{c} k_{0}, k_{0}=\frac{2 \pi}{\lambda}$. Here $\lambda$ is the wavelength of the incoming beam, and $\alpha_{c}$ is the convergence angle, an experimental parameter determining the spot size. In our simulations and experiments we make use of a $300 \mathrm{keV}$ electron beam, $k_{0}=3.19 \mathrm{pm}^{-1}$, with a semiconvergence angle $\alpha_{c}=4 \mu \mathrm{rad}$. Equation (12) reflects the experimental setup of a vortex beam that is generated by evenly illuminating the condenser aperture while giving it an extra vortex phase, after which the probe is formed in the far field of this aperture. For photons, this phase can be applied with, for instance, a spatial light modulator [34], while for electrons, this can be realized by placing the tip of a magnetized needle on the center of the condenser aperture [20]. In our experiment, however, the electron vortex beams are created by placing a fork hologram in the illumination system [18]. The condenser system then is used to project the FT of the hologram in the sample plane, and the vortex probe in Eq. (12) is obtained. For practical reasons, the triangular 
and multipinhole apertures were placed in the selected area plane which is conjugated to the image plane. Note that the experiments are done using an electron microscope, but the result is equally valid for optical vortex diffraction.

In Fig. 1, the real-space image of a vortex beam with $\ell=$ $-1, \ell=0$, and $\ell=+1$ centered on a multipinhole aperture that consists of five equidistant holes is shown together with the simulated and experimental diffraction patterns. Because of the fivefold symmetry of the aperture and the central position of the probe, the diffraction patterns all show fivefold symmetry. Also, because of Friedel's law, the $\ell=0$ beam shows extra twofold rotation symmetry, and the resulting diffraction pattern becomes tenfold symmetric. This symmetry is absent in the vortex-beam diffraction patterns. However, following Eq. (11), a twofold rotation symmetry can be observed between the $\ell=-1$ and $\ell=+1$ diffraction patterns. The same can be seen in Fig. 2, where $\ell=-1$ and $\ell=+1$ vortices are centered on a triangular aperture. Because of the threefold symmetry of the aperture and the central probe position, both diffraction patterns show threefold symmetry. Again, the two diffraction patterns are rotated $\pi$ rad with respect to each other.

In both examples, the vortex is centered on a high-symmetry point of the aperture, which ensures that the symmetry of the aperture is retained in all diffraction patterns. This ensures that the twofold rotational relation between the opposite vortex diffraction patterns is equivalent to a mirror operation or a rotation over $\pi / 5$ and $\pi / 3$ rad for the fivefold and threefold symmetric apertures, respectively. To show that the twofold rotation is independent of the symmetry of the beam position and symmetry of the aperture, in Fig. 3, we shift the vortex beam to a nonsymmetric position on the triangular aperture, thereby destroying the threefold symmetry. However, the twofold rotation relation between diffraction patterns of the opposite vortex beams remains present, as expected.

\section{DISCUSSION}

The EFL in Eq. (11) shows that diffraction patterns of oppositely charged vortices always are twofold symmetric with respect to each other when scattered on a real two-dimensional projected potential or two-dimensional apertures with real transmittance functions. The triangular aperture we used (Fig. 2) was already suggested to determine the topological charge of a vortex beam by Hickmann et al. [27]. By looking at the diffraction pattern, they were able to derive a rule for determining the magnitude of the OAM of the incoming beam, and they noted that diffraction patterns of opposite charge were always rotated by $\pi \mathrm{rad}$. Similarly, the pinhole aperture was proposed by Berkhout and Beijersbergen [13] as an alternative method to measure the topological charge of vortex beams. They also mention that, in the case of an odd number of holes, diffraction patterns of opposite charge mirror one another. Several other diffraction experiments with vortices were performed [29,30,35-39], and when a comparison with oppositely charged vortices was made, the same centrosymmetry between diffraction patterns was always observed.

In all these examples, however, the arguments to explain the symmetry between two opposite vortex-beam diffraction patterns relied on the symmetry of the specific apertures with respect to the center of the vortex (the studies we found on asymmetric apertures unfortunately do not compare opposite vortex-beam diffraction patterns). The EFL, however, shows that this symmetry is a fundamental property of vortex beams scattered on any real projected potential or two-dimensional apertures with real transmittance functions, independent of the symmetry of the scattering object.

Like Friedel's law, EFL is useful when studying crystallography with vortex beams. In previous work, for example, we proposed the use of electron vortex-beam diffraction to determine the handedness of chiral crystals [24], crystals that only differ by a mirror operation, starting from the idea that vortex beams themselves are chiral. However, by extending Friedel's law to vortex beams, it immediately becomes clear that the chirality cannot be learned directly from the projected potential of the crystal or the zeroth-order Laue zone (ZOLZ) in the diffraction pattern, within the kinematical approximation. Any chiral effect that would be seen with one vortex would differ by only a rotation of $\pi$ rad with the opposite vortex. Therefore the left-handed crystal would show the same ZOLZ as its right-handed mirror image when rotated $\pi$ rad along the beam's propagation direction. Looking at kinematically scattered electrons, any chiral effect must thus be searched for in the higher-order Laue zones that contain three-dimensional information about the crystal [40].

\section{CONCLUSION}

In this work, we derived an extension of Friedel's law that applies to vortex beams, whether it concerns an optical electron or any other particle beam. We show that when a vortex beam is diffracted on a two-dimensional scattering object, such as apertures, two diffraction patterns of vortex beams with opposite topological charge are always rotated $\pi \mathrm{rad}$ with respect to each other. This is independent of the symmetry of the scattering object and a fundamental property of vortex-beam scattering. Our findings also apply for diffraction on a crystal, when kinematical scattering between the zeroth-order Laue zone is considered. In general, this can be applied to x-ray diffraction but not to electron diffraction on crystals, which is mostly dominated by dynamical scattering.

We verified our analytical derivation with numerical simulations and showed experimental results for electron vortex beams scattered on multipinhole and triangular apertures. We compared our findings with observations made in the literature, where this effect mostly is explained using the symmetry of the scattering objects with respect to the center of the vortex beam. However, with our derivation and by looking at vortex beams scattering on nonsymmetric points of our apertures, we have shown that this symmetry is a more fundamental property of vortex-beam diffraction. The work presented here provides deeper understanding of vortex-beam diffraction, which in turn can be used to design new experiments to measure the topological charge of vortex beams with diffraction gratings or to study general vortex-beam diffraction.

\section{ACKNOWLEDGMENTS}

The authors acknowledge support from the FWO (Aspirant Fonds Wetenschappelijk Onderzoek - Vlaanderen) and the 
EU under the Seventh Framework Program (FP7) under a contract for an Integrated Infrastructure Initiative, Reference
No. 312483-ESTEEM2 and ERC Starting Grant No. 278510 VORTEX.
[1] M. G. Friedel, Sur les symétries cristallines que peut réveiler la diffraction des rayons Rontgen, C. R. Seances Acad. Sci., Ser. A 157, 1533 (1913).

[2] J. F. Nye and M. V. Berry, Dislocations in Wave Trains, Proc. R. Soc. London, Ser. A 336, 165 (1974).

[3] L. Allen, M. J. Padgett, and M. Babiker, IV The orbital angular momentum of light, Progress in Optics, 39, 291 (1999).

[4] K. Y. Bliokh, Y. P. Bliokh, S. Savel'ev, and F. Nori, Semiclassical Dynamics of Electron Wave Packet States with Phase Vortices, Phys. Rev. Lett. 99, 190404 (2007).

[5] C. W. Clark, R. Barankov, M. G. Huber, M. Arif, D. G. Cory, and D. A. Pushin, Controlling neutron orbital angular momentum, Nature (London) 525, 504 (2015).

[6] V. Y. Bazhenov, M. V. Vasnetsov, and M. S. Soskin, Laser beams with screw dislocations in their wavefronts, JETP Lett. 52, 429 (1990).

[7] Z.-P. Luo, Y.-L. Sun, and K.-N. An, An optical spin micromotor, Appl. Phys. Lett. 76, 1779 (2000).

[8] H. He, M. E. J. Friese, N. R. Heckenberg, and H. RubinszteinDunlop, Direct Observation of Transfer of Angular Momentum to Absorptive Particles from a Laser Beam with a Phase Singularity, Phys. Rev. Lett. 75, 826 (1995).

[9] M. E. J. Friese, H. Rubinsztein-Dunlop, J. Gold, P. Hagberg, and D. Hanstorp, Optically driven micromachine elements, Appl. Phys. Lett. 78, 547 (2001).

[10] G. Foo, D. M. Palacios, and G. A. Swartzlander, Optical vortex coronagraph, Opt. Lett. 30, 3308 (2005).

[11] G. Swartzlander and R. Hernandez-Aranda, Optical Rankine Vortex and Anomalous Circulation of Light, Phys. Rev. Lett. 99, 163901 (2007).

[12] E. Serabyn, D. Mawet, and R. Burruss, An image of an exoplanet separated by two diffraction beamwidths from a star, Nature (London) 464, 1018 (2010).

[13] G. C. G. Berkhout and M. W. Beijersbergen, Method for Probing the Orbital Angular Momentum of Optical Vortices in Electromagnetic Waves from Astronomical Objects, Phys. Rev. Lett. 101, 100801 (2008).

[14] Structured Light and Its Applications: An Introduction to PhaseStructured Beams and Nanoscale Optical Forces, edited by D. L. Andrews (Elsevier, Cambridge, 2011).

[15] J. Wang, J.-Y. Yang, I. M. Fazal, N. Ahmed, Y. Yan, H. Huang, Y. Ren, Y. Yue, S. Dolinar, M. Tur, and A. E. Willner, Terabit freespace data transmission employing orbital angular momentum multiplexing, Nat. Photonics 6, 488 (2012).

[16] S. Roychowdhury, V. K. Jaiswal, and R. Singh, Implementing controlled NOT gate with optical vortex, Opt. Commun. 236, 419 (2004).

[17] M. Uchida and A. Tonomura, Generation of electron beams carrying orbital angular momentum, Nature (London) 464, 737 (2010).

[18] J. Verbeeck, H. Tian, and P. Schattschneider, Production and application of electron vortex beams, Nature (London) 467, 301 (2010).
[19] L. Clark, A. Béché, G. Guzzinati, A. Lubk, M. Mazilu, R. Van Boxem, and J. Verbeeck, Exploiting Lens Aberrations to Create Electron-Vortex Beams, Phys. Rev. Lett. 111, 064801 (2013).

[20] A. Béché, R. Van Boxem, G. Van Tendeloo, and J. Verbeeck, Magnetic monopole field exposed by electrons, Nat. Phys. 10, 26 (2013).

[21] R. Juchtmans, L. Clark, A. Lubk, and J. Verbeeck, Spiral phase plate contrast in optical and electron microscopy, Phys. Rev. A 94, 023838 (2016).

[22] J. Verbeeck, H. Tian, and G. Van Tendeloo, How to Manipulate Nanoparticles with an Electron Beam?, Adv. Mater. 25, 1114 (2013)

[23] E. Karimi, L. Marrucci, V. Grillo, and E. Santamato, Spin-toOrbital Angular Momentum Conversion and Spin-Polarization Filtering in Electron Beams, Phys. Rev. Lett. 108, 044801 (2012).

[24] R. Juchtmans, A. Béché, A. Abakumov, M. Batuk, and J. Verbeeck, Using electron vortex beams to determine chirality of crystals in transmission electron microscopy, Phys. Rev. B 91, 094112 (2015).

[25] R. Juchtmans and J. Verbeeck, Orbital angular momentum in electron diffraction and its use to determine chiral crystal symmetries, Phys. Rev. B 92, 134108 (2015).

[26] R. Juchtmans and J. Verbeeck, Local orbital angular momentum revealed by spiral phase plate imaging in transmission electron microscopy, Phys. Rev. A 93, 023811 (2016).

[27] J. M. Hickmann, E. J. S. Fonseca, W. C. Soares, and S. ChávezCerda, Unveiling a Truncated Optical Lattice Associated with a Triangular Aperture Using Light's Orbital Angular Momentum, Phys. Rev. Lett. 105, 053904 (2010).

[28] G. Guzzinati, L. Clark, A. Béché, and J. Verbeeck, Measuring the orbital angular momentum of electron beams, Phys. Rev. A 89, 025803 (2014).

[29] C.-S. Guo, S.-J. Yue, and G.-X. Wei, Measuring the Orbital Angular Momentum of Optical Vortices Using a Multipinhole Plate, Appl. Phys. Lett. 94, 231104 (2009).

[30] L. Shi, L. Tian, and X. Chen, Characterizing topological charge of optical vortex using non-uniformly distributed multi-pinhole plate, Chin. Opt. Lett. 10, 120501 (2012).

[31] L. Clark, A. Béché, G. Guzzinati, and J. Verbeeck, Quantitative measurement of orbital angular momentum in electron microscopy, Phys. Rev. A 89, 053818 (2014).

[32] M. De Graef, Introduction to Conventional Transmission Electron Microscopy (Cambridge University Press, Cambridge, 2003).

[33] M. Born and E. Wolf, Principles of Optics: Electromagnetic Theory of Propagation, Interference and Diffraction of Light, 7th ed. (Cambridge University Press, New York, 1999).

[34] Z. Bouchal, R. Čelechovský, and V. Kollárov, Nondiffracting and vortex beams generated by spatial light modulator, Proc. SPIE 5945, 59450F (2005).

[35] H. I. Sztul and R. R. Alfano, Double-slit interference with Laguerre-Gaussian beams, Opt. Lett. 31, 999 (2006). 
[36] M. Mazilu, A. Mourka, T. Vettenburg, E. M. Wright, and K. Dholakia, Simultaneous determination of the constituent azimuthal and radial mode indices for light fields possessing orbital angular momentum, Appl. Phys. Lett. 100, 231115 (2012).

[37] D. P. Ghai, P. Senthilkumaran, and R. S. Sirohi, Single-slit diffraction of an optical beam with phase singularity, Opt. Lasers Eng. 47, 123 (2009).

[38] R. Liu, J. Long, F. Wang, Y. Wang, Pei Zhang, Hong Gao, and Fuli $\mathrm{Li}$, Characterizing the phase profile of a vortex beam with angular-double-slit interference, J. Opt. 15, 125712 (2013).

[39] Q. S. Ferreira, A. J. Jesus-Silva, E. J. S. Fonseca, and J. M. Hickmann, Fraunhofer diffraction of light with orbital angular momentum by a slit, Opt. Lett. 36, 3106 (2011).

[40] In this case, it is easy to show that the HOLZ of an $\ell$ vortex beam is rotated $\pi \mathrm{rad}$ with respect to the $-\ell$ vortex when, at the same time, the potential is mirrored along the plane perpendicular to the propagation of the vortex beam. 